\title{
FEA Assisted Design and Structural Research on Vertical Axis Wind Turbine Rotor
}

\author{
Siddhan Sivakumar, Sathyabalan P.
}

\begin{abstract}
The scope of the project is to perform finite element analysis of the Vertical axis wind turbine rotor assembly.Blade, arm, connectors and strings assembly of rotor are considered for analysis and to study the static behaviour at the different loading conditions. Total vertical load of $132 \mathrm{~kg}$ self-weight is acting downward direction to the structure. Considered that rotor will be rotating at maximum speed (RPM) aerodynamic load with self weight conditions. Due to higher angular velocity (154 RPM) blades will be subjected to great centrifugal force. Along with the centrifugal load rotor also will get subjected to fluctuating aero dynamic loads. In analysis it is considered as the rotor in stationary position and subjected to the maximum drag force at $52(\mathrm{~m} / \mathrm{s})$ wind velocity at azimuth value of $90^{\circ}$. SOLID92 is used for the 3-D modelling of solid structures. The maximum stress value obtained from the analysis is 183.1 $\mathrm{N} / \mathrm{mm} 2$ for the loading and boundary conditions.
\end{abstract}

Keywords-Keywords: FEA, VAWT, Blade

\section{INTRODUCTION}

The interest in harvesting renewable energy, especially wind energy, in offshore as well as in the urban environment has significantly increased in recent years [1][2][3]. Vertical axis wind turbines(VAWT) are receiving considerable attention in recent years as they are able to capture the wind from any direction. They are easy to install, easy to transport, cheaper to build and maintain, and quite safe to humans and birds. They are especially suitable for urban areas or areas with extreme weather. However, they are less efficient than horizontal axis turbines because only one blade is active at a time, and VAWTs also generate a relatively high degree of vibration and noise pollution.

Wind turbines can be classified into two types based on its rotational axis direction: HAWT (horizontal axis wind turbine) and VAWT (vertical axis wind turbine). HAWT is better suited for large-scale energy generation while VAWT is easier to install, omni-directional and has a low noise level [4]. From the perspective of self starting capability and turbine efficiency,VAWT can be classified into two types, the drag type Savonius and lift type Darrieus VAWT [5]. The former has a good self-starting capability but with low self-starting capability. The H-rotor Darrieus VAWT has a relatively simple design comprised of straight blades of uniform airfoil chord and blade radius, and is becoming more and more attractive due to its unique advantages such

Revised Manuscript Received on August 19, 2019.

SiddhanSivakumar, Department of Mechanical Engineering Kumaraguru College of Technology Coimbatore, T.N, India-641049.(Email:sivakumar.siddhan.mec@kct.ac.in)

Sathyabalan P, Department of Mechanical Engineering Kumaraguru College of TechnologyCoimbatore, T.N, India,641049.(Email:sathyabalan.p.mec@kct.ac.in) efficiency, while the latter has a better efficiency but a poor

as easy installation, low noise level and low cost different types of wind loads, locations and its directions are illustrated as shown in Fig .1

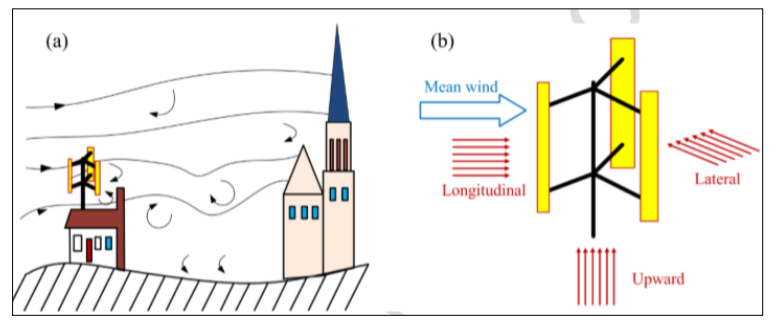

Fig. 1 Wind loads inVAWT rotor Assembly[6]

In the vertical axis wind turbine thrust forces are absent. Drag and lift forces are main components along with self weight. Drag and lift forces are mainly depend on blade profile and azimuth of rotor. Azimuth of rotor gives the position of blade at selected time. Angle of azimuth ranges from $0-360^{\circ}$. The parameters that can affect the magnitude of force are:

- Azimuth of turbine (rad)

- Camber height of the blade profile (m)

- Camber location on mean line of the blade's chordlength $(\mathrm{m})$

- Thickness of the blade (m)

- Aspect ratio of the blade (m)

In this paper, finite element analysis of the Vertical axis wind turbine rotor assembly is studied.Blade, arm, connectors and strings assembly of rotor are considered for analysis and to study the static behaviour at the different loading conditions. Total vertical load of $132 \mathrm{~kg}$ self-weight is acting downward direction to the structure.

\section{MODEL INFORMATION}

\section{A. Finite Element model}

The rotor is symmetric about the axis, considering this only half of the rotor is modelled. Boundary conditions and wind load conditions applied according to the relevant calculations. Fig. 2 shows the meshed model of the Rotor assembly.SOLID92 elements are used to mesh the model and the details of elements used are listed in the Table 1. The Finite Element model of the full assembly including connectors and strings is given in Fig. 2.

\section{Published By:}

Blue Eyes Intelligence Engineering 
TABLE I. ELEMENT TYPE USED FOR VAWT ROTOR ASSEMBLY

\begin{tabular}{|c|l|c|}
\hline S. No & Part & Type of Element \\
\hline 1 & Blade & Solid 92 \\
\hline 2 & Arm & Solid 92 \\
\hline 3 & Connectors & Cerig\&MASS21 (dummy) \\
\hline 4 & Strings & BEAM4 \\
\hline
\end{tabular}

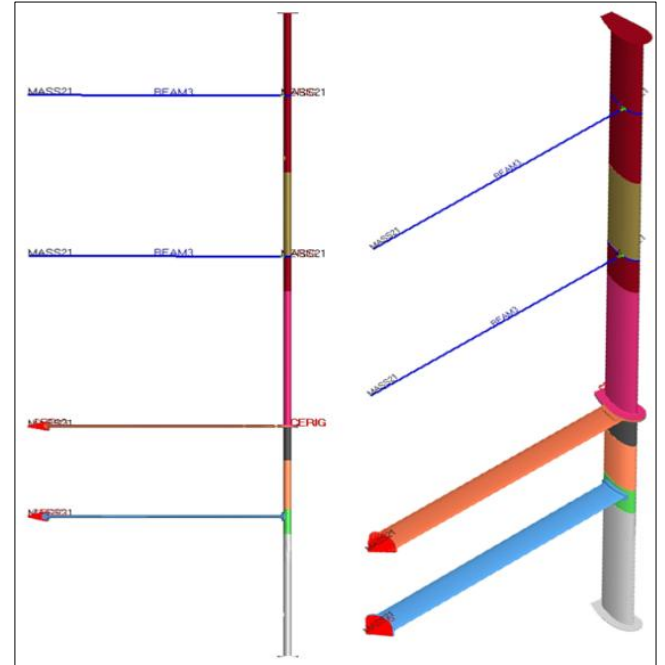

Fig. 2 Finite Element Model of VAWT rotor Assembly

\section{B. Boundary conditions}

SOLID92 is used for the 3-D modeling of solid structures. It is 3-D 10-Node Tetrahedral Structural Solid as given in Fig. 3. The element is defined by ten nodes having three degrees of freedom at each node: translations in the nodal $x$, $\mathrm{y}$, and $\mathrm{z}$ directions.

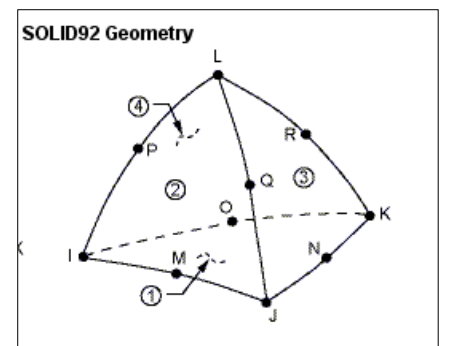

Fig. 3 Element used for Meshing ofVAWT rotor Assembly

\section{Loading conditions for VAWT rotor Assembly}

a) Loading condition 1:Total vertical load of $132 \mathrm{~kg}$ self weight is acting downward direction to the structure. Considered that rotor will be rotating at maximumspeed (RPM) . Due to higher angular velocity (154 RPM) blades get subjected to great centrifugal force. In analysis speed is applied globally on the rotor assembly to allow rotation in $\mathrm{Y}$-axis rotational degrees of freedom is left free rest of five degrees of freedom are arrested. The loading and boundary conditions details are shown in Fig. 4, Fig. 5 and Fig. 6

b) Loading condition 2:Along with the centrifugal load rotor also will be subjected to fluctuating Aero dynamic loads. When the rotor is in rotation the drag and lift forces will be lesser but the worst case is at maximum wind velocity and maximum drag condition
Maximum drag force occurs when the rotor exposed area at azimuth value of $90^{\circ}$ and $270^{\circ}$. In analysis it is considered as the rotor in stationary position and subjected to the maximum drag force at $52(\mathrm{~m} / \mathrm{s})$ wind velocity at azimuth value of $90^{\circ}$.

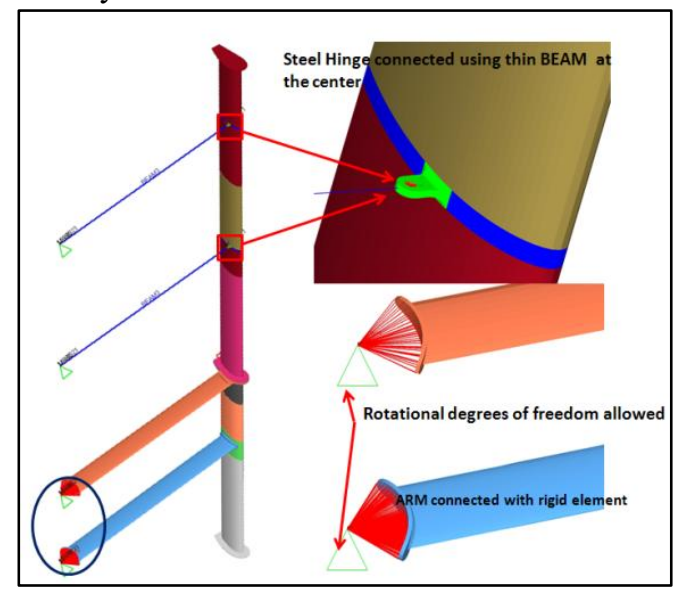

Fig. 4 Boundary conditions considered for VAWT rotor Assembly

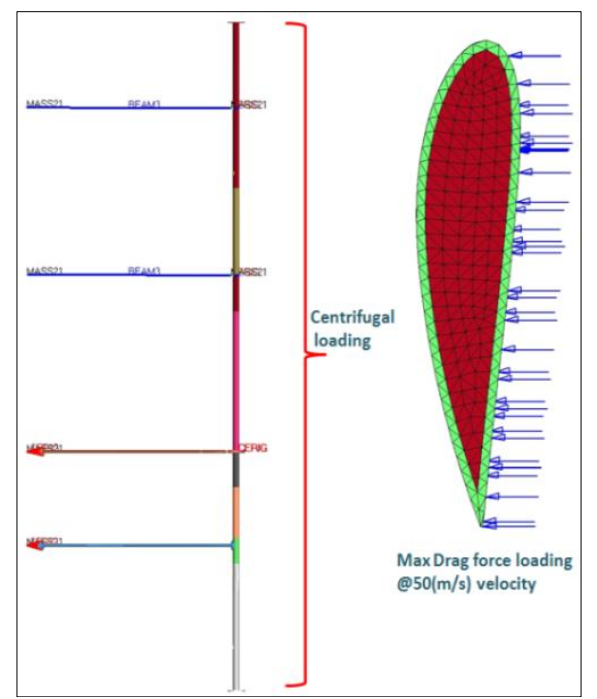

Fig. 5 Loading details for VAWT rotor Assembly

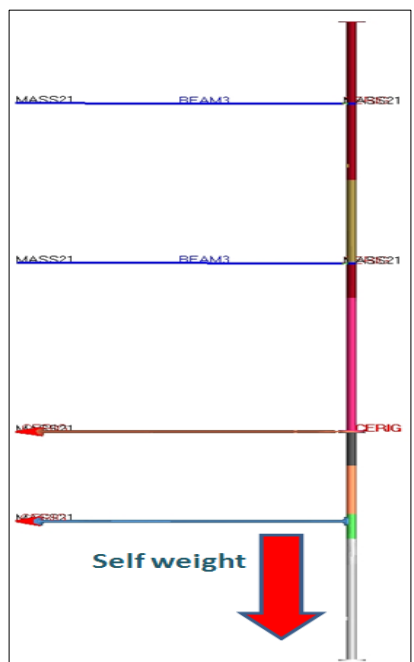

Fig. 6. Loading details (Self weight)

Published By: 


\section{D.Material Properties}

Material used in this analysis are plastic fiber and steel for the different parts and its properties are given in the Table 2

Table 2: Material Properties

\begin{tabular}{|c|c|c|c|c|c|}
\hline Part & Material & $\begin{array}{c}\text { Young's modulus } \\
\text { (N/mm2) }\end{array}$ & Poisson's Ratio & $\begin{array}{c}\text { Density } \\
\text { (kg/m3) }\end{array}$ & $\begin{array}{c}\text { Ultimate Tensile } \\
\text { Strength } \\
(\mathbf{M P a})\end{array}$ \\
\hline Blade & Carbon Fiber & $2.2 \mathrm{e} 5$ & 0.2 & 1770 & 3500 \\
\hline Inside the Blade & $\begin{array}{c}\text { PUF(polyurethane } \\
\text { foam) }\end{array}$ & $0.41 \mathrm{E} 5$ & 0.3 & 1570 & 313 \\
\hline Arm & $\begin{array}{c}\text { FRP(Fiber reinforced } \\
\text { plastic) }\end{array}$ & $0.7 \mathrm{e} 5$ & 0.25 & 1750 & 5650 \\
\hline Hinge & Steel & $2.1 \mathrm{e} 5$ & 0.33 & 7890 & 370 \\
\hline Impregnated rods & FRP & $0.7 \mathrm{e} 5$ & 0.25 & 1750 & 5650 \\
\hline
\end{tabular}

\section{E. Design Methodology}

Self weight $132 \mathrm{~kg}$ self-weight is acting on the structure. Due to higher angular velocity (154 RPM), centrifugal forces acting on the blades are of high magnitudes. Fluctuating aero dynamic loads will also be acting on the rotor. When the rotor is in rotation the drag and lift forces will be lesser but the worst case is at maximum wind velocity and maximum drag condition.

\section{RESULTS AND DISCUSSION}

\section{A. Displacement plot}

The displacement plots obtained from the analysis is as follows, The Vertical displacement plot, linear displacement plot and Lateral displacement plot are shown in Figure 7, 8\&9 respectively

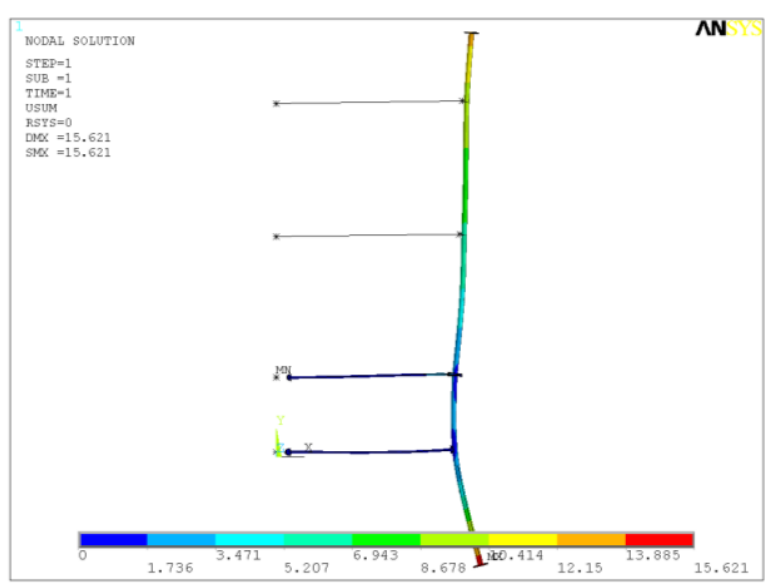

Fig. 7. Resultant displacements of assembly (Usum) in mm

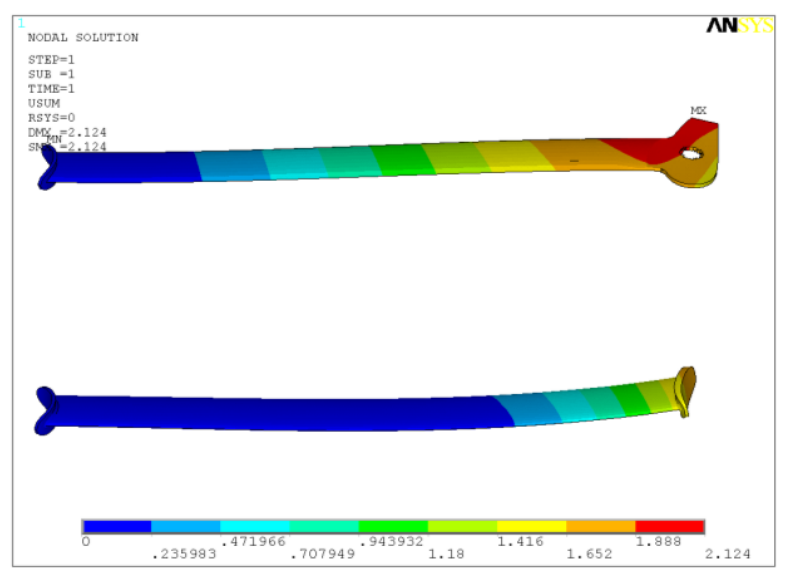

Fig. 8. Resultant displacement of rotor (Usum) in $\mathbf{m m}$

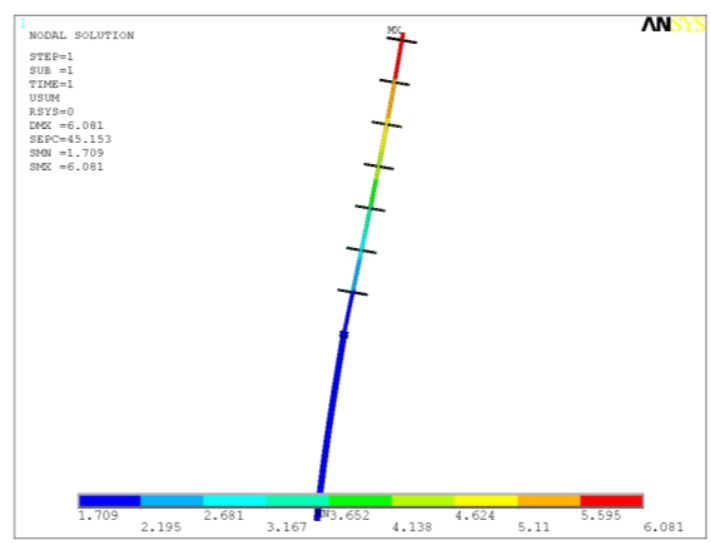

Fig. 9. Displacement plots ofRods inserted in the $\operatorname{Blade}(\mathbf{m m})$

\section{B.Stress plot}

Figure 10-11 its Shows vonmises stress distribution behaviour in the VAWT rotor and its assembly. From the figure its observed that max stresses induced in the different parts like hinge, blade, arm and blade packing lie within the allowable stress limits as shown in table 2. 


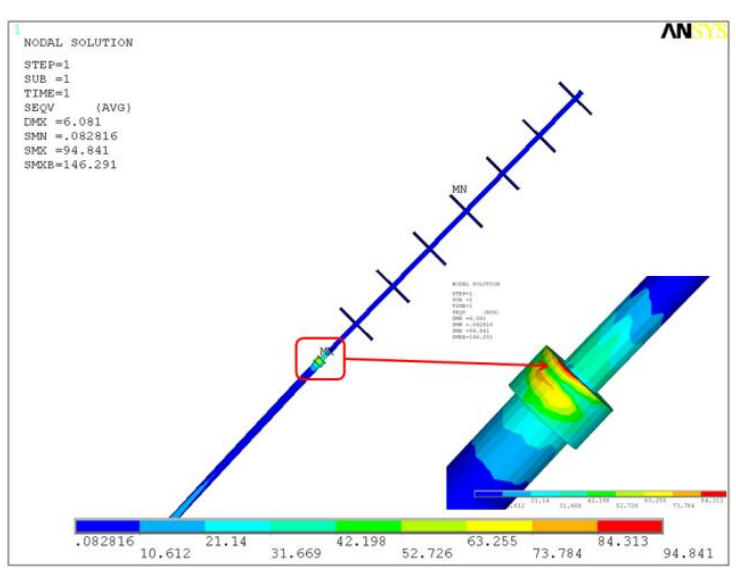

Fig. 10.VonMises stress plot of hinge (MPa)

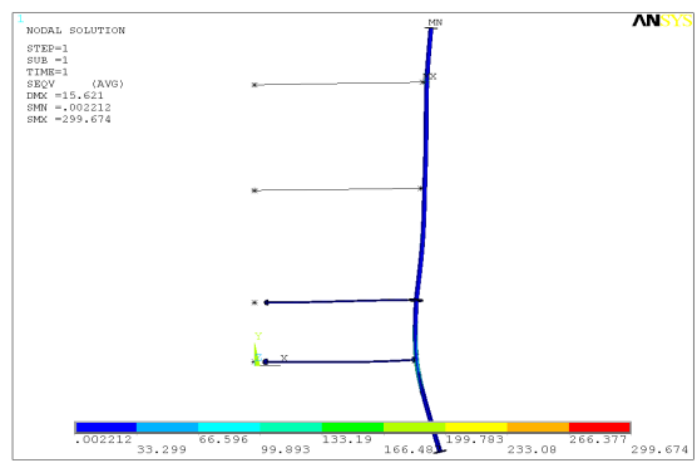

Fig. 11 :VonMises stress plot of assembly (MPa)

\section{CONCLUSIONS}

- Maximum Vonmises Stress of assembly 299.6 MPa is obtained at hinge location and having factor of safety of 1.03.FOS can be increased making more interface between hinge and blade

- Stresses in other components are lesser, but the FRP , PUF and carbon fiber materials are not having yielding nature. They fails when the material subjected to Ultimate Tensile Stress.The values and factor of safety are indicated depend on UTS values.

- The Maximum displacement obtained from the analysis is $15.622 \mathrm{~mm}$ in horizontal axis (X) for above loading \& boundary conditions. Resultant displacement can be reduced by making the overhanging portion lesser at the bottom section of the blade.

- Due to self weight it is having very small deflection of $2.5(\mathrm{~mm})$ in vertical axis $(\mathrm{Y})$.

\section{REFERENCES}

1. S. G. Gawade and D. S. Patil, "Comparative study of a single stage Savonius with a combined Savonius-three bladed Darrieus," International Journal for Technological research in Engineering, vol. 2, issue 6, February 2015

2. S. J. Savonius, "The S - rotor and its application," Mech Eng. vol. 53, pp. 333-338, 1931

3. Wilcox, David C, "Turbulence Modeling for CFD", Second edition, Anaheim, DCW Industries, 1998.

4. Nisi, K., B. Nagaraj, and A. Agalya. "Tuning of a PID controller using evolutionary multi objective optimization methodologies and application to the pulp and paper industry." International Journal of Machine Learning and Cybernetics (2018): 1-11.

5. M. H. Ali, "Experimental comparison study for Savonius wind turbine of two and three blades at low wind speed," International Journal of Modern Engineering Research, vol. 3, issue 5, 2013
6. Rezaeiha A, Kalkman I, Blocken B. Effect of pitch angle on power performance and aerodynamics of a vertical axis wind turbine. Applied Energy. 2017; 197:132-50

7. Tummala A, Velamati RK, Sinha DK, Indraja V, Krishna VH. A review on small scale wind turbines. Renewable and Sustainable Energy Reviews. 2016;56:1351-71. 